\title{
An examination of the semantic adjustment hypothesis of contrast effects in loudness judgments
}

\author{
LAWRENCE E. MELAMED and WENDY WAUGH \\ Kent State University, Kent, Ohio 44242
}

\begin{abstract}
Subjects were presented a series of soft tones for loudness judgments using a category rating scale. After several blocks of soft tones, sets of loud tones were introduced to be presented along with the soft tones. This shift in the level of the tones to be judged was employed to examine any contrast effects (shifts in judgment) for the soft tones in comparison with earlier judgments. The loud series was presented only on the 1st postshift trial block, or on the 1st and 5th such trial blocks, or on the 1st, 5th, and 9th blocks, or on all 12 postshift trial blocks. The form of the contrast effects obtained across these groups was examined for its support of Steven's (1958) semantic adjustment hypothesis of category judgments.
\end{abstract}

When an anchoring stimulus is introduced outside one end of a series of stimuli being judged, a displacement of the judgments of these stimuli away from the anchor is produced. This is the contrast effect commonly found in studies using category or comparative rating scales. According to adaptation-level theory (Helson, 1964), the contrast effect represents a shift in the sensory magnitude of the stimuli. The mechanism assumed to underlie this change is the current adaptation level of the subject, which is represented by the stimulus value that appears neutral or indifferent. The distance of a stimulus from this neutral point determines its sensory magnitude. The effect of presenting an anchoring stimulus is to pull the adaptation level toward the anchor's value.

Alternative interpretations of the contrast effect have focused on judgmental factors. Range-frequency theory (Parducci, 1965) proposes two major assumptions to explain contextual effects with category judgments. The first is that stimulation established a subjective range, which the subject divides into subranges corresponding to the categories available for judgment. It is the extreme stimuli, the endpoints, that determine the range. When extreme stimuli are presented outside the series being judged, the subjective range broadens to include the anchors. It is also assumed that categories tend to be used with equal frequency. Contrast effects reflect the position of the series stimuli relative to the extended range. When the anchor is removed, ratings shift back to preanchor values due to the tendency of subjects to use the response categories with equal frequency.

Another explanation for contrast effects that is appropriate when category rating scales are used is that

Requests for reprints should be sent to Lawrence E. Melamed, Department of Psychology, Kent State University, Kent, Ohio 44242. of Stevens (1958). He argues that these effects simply represent "semantic adjustments," a change in modulus for a category when the stimulus range is extended. Thus in a loudness judgment task, a tone that was judged medium in an original "soft" set may only be judged soft in an extended stimulus range. The categories have referents that are adjusted for the particular set of stimuli being judged.

It would seem that one way of evaluating Stevens' (1958) hypothesis in comparison with those of Helson (1964) and Parducci (1965) within the framework of a loudness judgment task would be to present, for example, a loud-tone set, the "anchor," in a limited manner and then determine the stability of any subsequent changes in the judgment of a set of soft tones. This is accomplished in the present experiment in the following manner: After six preliminary blocks of soft tones, an interspersed set of loud and soft tones occurs only on Block 7 for one group, on Blocks 7 and 11 for another, and on Blocks 7, 11, and 15 for a third. Control groups judge soft tones for 18 trial blocks or soft and loud tones for Blocks 7-18. Both adaptation-level and range-frequency theory would predict decrements in the loudness judgments of the soft tones that would be related to the proximity and frequency of presentation of the loud-tone anchors. The semantic adjustment hypothesis would lead to the expectation that the contrast effect would simply "kick in" at some point (e.g., upon the first or second presentation of the anchor) and then the loudness judgments of the soft tones would stabilize at some reduced level. The contrast effect would not be expected to either increase or decrease after its initial appearance.

\section{METHOD}

\section{Subjects}

Seventy-five female undergraduates with normal hearing thresholds were used in the experiment. The subjects were 
recruited from introductory psychology sections and received course credit for their participation.

\section{Stimuli and Apparatus}

The stimuli consisted of two series of four $1,000-\mathrm{Hz}$ tones differing in intensity by $5-\mathrm{dB}$ intervals. The soft series (S) contained 30-, 35-, 40-, and 45-dB SL tones. The loud series (L) included tones of $75,80,85$, and $90 \mathrm{~dB}$ SL. Each tone was $1 \mathrm{sec}$ in duration, with a $10 \mathrm{sec}$ interstimulus interval. The tones were presented monaurally to subjects seated inside an audiometric test chamber.

\section{Design and Procedure}

Fifteen subjects were randomly assigned to each of five groups. All subjects made category judgments of the soft-tone series for six blocks of trials (preshift judgments) in which each of the four soft tones occurred once in each of the blocks. The control group (S-S) continued to judge the soft tones for 12 additional blocks of trials. In addition to the soft tones, the experimental groups judged the loud tones on certain of the postshift blocks of trials. Group $(S-S+L 7)$ received the loud and soft series together only in the seventh block of trials, the first postshift one. Group $(S-S+L 7,11)$ judged the combined series on one-sixth of the postshift trial blocks, 7 and 11. Subjects in the $(S-S+L 7,11,15)$ group received the additional loud series on Trial Blocks 7, 11, and 15. The fifth group $(S-S+L)$ judged both soft and loud tones on all postshift blocks. When both loud and soft tones were presented, stimuli were randomized over the two series.

Subjects were instructed to rate the loudness of each tone using categories coded from 1 to 9 (very very soft $=1$ ). The rating scale was present throughout the session to ensure that the subject was aware of all possible judgments. Each subject recorded her own responses on a data sheet containing blanks numbered from 1 to 120 . Previous responses were kept covered by a cardboard stencil manipulated to reveal only one response blank at a time.

\section{RESULTS}

Loudness judgments on the 12 postshift blocks of trials were analyzed using a 5 by 4 by 12 mixed-design analysis of variance in which the between-subjects factor was treatment group membership and the withinsubjects factors were the four soft tones and the 12 judgment trials of each.

For the sake of parsimony, the significant Groups by Trials effect $[F(44,770)=3.84, p<.001]$ will be the major focus of this section. This interaction is the basis for determining whether or not there is any difference in the structure and pattern of the contrast effects for the various experimental groups. The group means for each trial are presented graphically in Figure 1. The pattern of the contrast effects can be discerned there. The central question concerns the comparability in the patterns of judgment subsequent to single and repeated presentations of the loud-tone series in the experimental groups.

A significant contrast effect was found for all experimental groups on Trial 8 following presentation of the loud tones on Trial 7. However, on Trials 9 and 10, there was an immediate increase in the ratings of the soft tones for Groups $(\mathrm{S}-\mathrm{S}+\mathrm{L} 7),(\mathrm{S}-\mathrm{S}+\mathrm{L7}, 11)$, and $(S-S+L 7,11,15)$, although all remained significantly

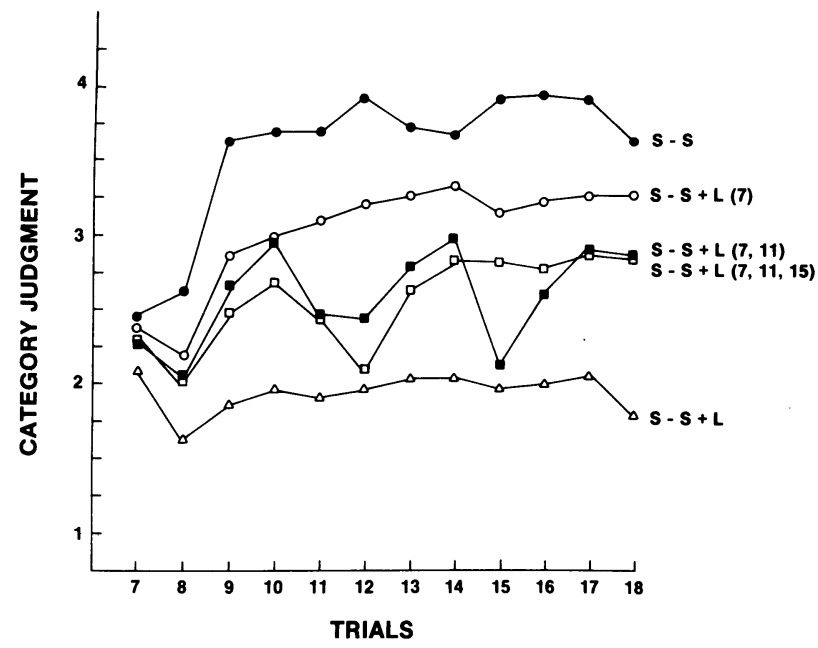

Figure 1. Loudness judgments for each group for the 12 postshift trials averaged over the soft-tone set.

below the controls (S-S). Beginning with Trial 11, and for all of the following postshift trials, the $(S-S+L 7)$ group was not significantly different from the control group in their ratings of the soft tones. This group's recovery was fairly complete, then, within four trials.

Groups $(\mathrm{S}-\mathrm{S}+\mathrm{L} 7,11)$ and $(\mathrm{S}-\mathrm{S}+\mathrm{L} 7,11,15)$ showed significant contrast effects on Trial 12 following their second exposure to the loud series on Trial 11 . Concentrating on Group $(\mathrm{S}-\mathrm{S}+\mathrm{L7}, 11)$, Figure 1 shows that the second contrast effect was comparable in size to the first. Further, the ratings in this group increased significantly on Trial 13 and remained consistent over the rest of the postshift trials. However, they did remain significantly below those of the controls (S-S) on all trials, although not significantly below those of Group (S-S + L7). The third presentation of loud tones again produced a significant contrast effect in the $(\mathrm{S}-\mathrm{S}+\mathrm{L} 7,11,15)$ group. However, this group's recovery from this effect was very rapid, being relatively complete on the very next trial. It should be pointed out that the judgments of this group were significantly lower than those of Group (S-S) on all postshift trials but were not significantly different from those of $(S-S+L 7)$ and $(S-S+L 7,11)$. The judgments of Group $(S-S+L)$ were always significantly lower than the (S-S) group, and likewise for Group (S-S + L7) after Trial 9. The $(S-S+L)$ group was generally not significantly different from $(S-S+L 7,11)$ and $(S-S+L 7,11,15)$ on the trial in which the loud tones were presented or on the next one.

\section{DISCUSSION}

The general pattern of the data is that presentation of the loud tones causes a decrement in the judged loudness of the soft tones. On one hand, the effect seems short-lived. Group $(S-S+L 7)$ judgments of the soft tones return to the level of the control group, Group (S-S), within a few trials. On the other 
hand, there is some carry-over effect. When contrast effects are subsequently produced on Trial 11 in Group $(S-S+L 7,11)$ and on Trials 11 and 15 in Group $(S-S+L 7,11,15)$, the subjects' judgments do not return to the level of the control group. Within a few trials, they are not significantly different from each other or from Group $(S-S+L 7)$. The second presentation of the loud tones in these groups does seem, therefore, to have some added effect on the subjects' judgment, although the overall pattern of effect seems rather similar within each four-block sequence in the experimental groups. With regard to this last point, it should be noted that the contrast effect is of pretty constant size wherever it occurs. The judgments of the soft tones in Groups $(S-S+L 7)$, $(S-S+L 7,11)$, and $(S-S+L 7,11,15)$ are not significantly different from those in Group $(S-S+L)$ on those trials on which loud tones are presented to both groups. One further aspect of the present data would seem important in evaluating the theories of contrast effects. This is the apparently faster recovery from the contrast effect in Group $(S-S+L 7,11,15)$ that occurred upon the last presentation of the loud tones, on Trial Block 15. Recovery was complete by Trial 16 .

The semantic adjustment hypothesis of Stevens (1958) can handle the constant size of the contrast effect in the experimental groups of the present experiment. The supposition would be that the subjects simply change the modulus for a category whenever the stimulus range is extended. The only difficulty for this approach, which is troublesome, is why any shift in modulus would be so transient in the experimental groups. Once the subjects hear the loud-tone set, and certainly after a second or third exposure to it, it would seem reasonable to expect it to continue to influence the judgments of the soft tones for more than two trials. It is also not evident why the contrast effect would decrease so quickly over these few trials. The theory that would seem to fit these data the least would be the range-frequency theory. The theory would have to accept the proposition that there is a very fast-acting redistribution of category usage that stabilizes very quickly and fades quickly (i.e., within three trials of the presentation of the loud tones in the experimental groups).

Probably the most straightforward approach in explaining the current data would be that obtained through the use of adaptation-level theory. The consistency in the pattern of the contrast effects for the experimental groups could be handled by assuming that there is a strong recency effect in the contribution of stimuli to the adaptation level. The loud tones shift the adaptation level higher on Trials 7,11 , and 15 , but it goes down just as quickly on subsequent trials in the four-trial sequences. The quicker return to baseline in Group $(\mathrm{S}-\mathrm{S}+$ L7,11,15) after Trial 15 would not seem inconsistent with a recency effect interpretation.

\section{REFERENCES}

Helson, H. Adaptation-level theory. New York: Harper \& Row, 1964.

Parducci, A. Category judgment: A range-frequency model. Psychological Review, 1965, 72, 407-418.

Stevens, S. S. Adaptation level vs. the relativity of judgment. American Journal of Psychology, 1958, 71, 633-646.

(Received for publication September 21, 1981.) 